\title{
The effect of Nursing Intervention Protocol About Laparoscopic Urological Surgery on Nurse's Performance and Patient Outcome
}

\author{
Eslah Abd Elmonam Mohamed ${ }^{1}$, Zienab Abd Ellatief Muhammad $^{2}$, Ahmed Serag Safwat ${ }^{3}$ \& Sahar Ali \\ AbdElmohsen ${ }^{4}$. \\ 1. Nursing Specialist at Urological Operating Room Urology \& Nephrology Hospital, at Assiut University, Egypt. \\ 2. Professor of Medical Surgical Nursing, Faculty of Nursing -Assuit University, Egypt. \\ 3. Professor of Urology, Faculty of Medicine -Assuit University, Egypt. \\ 4. Lecturer of Medical Surgical Nursing, Faculty of Nursing, Assiut University, Egypt.
}

\begin{abstract}
Laparoscopy is a surgical procedure that allows for visualization of the abdominal cavity through a small incision. The aim of this study was to assess the effect of nursing Intervention Protocol about laparoscopic urological surgery on nurse's Performance and Patient outcome. Research design: A Quasi experimental research design was used to conduct this study. Setting: The study was carried out at urological operating room in urology and nephrology hospital. Sample: All available nurses (17) and 60 patients were included in this study. Tools of data collection were nurse's knowledge assessment questionnaire method, nurse's practice observational checklist, and Patient assessment sheet. Results: A positive correlation between nurses' knowledge and practice regarding urologic abdominal laparoscopic surgery. There was a statistically significant difference between both times of follow-up regarding incidence of complications. Conclusion: The nurse's knowledge and performance about laparoscopic surgery were limited. Recommendations: Improve level of professional nurses 'performance through attending scientific meetings, conferences and well-structured training programs should be offered to the nurses for maintain high-quality of care.
\end{abstract}

\section{Keywords: Laparoscopy, Nurses Performance, Patient Outcome \&Urology.}

\section{Introduction}

Laparoscopy is a surgical technique in which a thin fiber optic cable system is passed through small cuts in the skin to gain access to the target surgical site without making a big skin incision, as is required in a traditional open surgery. This technique is performed using a video camera that delivers visuals of the surgical site to a television monitor that the surgeon uses as a guide. Due to this, all procedures performed using laparoscopy are referred to as "minimally invasive" or "keyhole surgery.

\section{(Bonjer, et al.2015)}

Nurses play an important role in maintaining the health and well-being of patients. The nurse has to understand the principles of minimally invasive surgery and identify the function of the different laparoscopic equipment and instruments. The nurses have to know how to troubleshoot different scenarios in equipment and instruments failure. Also she should be familiar with the principles of sterilization and disinfection in laparoscopy set, to be able to identify, arrange and set up the various laparoscopic equipment, instruments as well to be able to prepare the preparation of the operating room, the nurse must demonstrate her knowledge, and be an expert technologist in dealing with malfunctions (Thomas, 2011)
Each one of the nursing team "nurse coordinator, scrub-nurse and circulating-nurse" has a certain job description to ensure maximum patient's safety and surgical efficiency. It's important to understand the complications that may occur, as with any surgical procedure. The nurse also must demonstrate her knowledge regarding the advantages and disadvantages of laparoscopic surgery to properly anticipate unintended complications. (Kang, 2016)

\section{Significance of study}

Nurses play an important role and do greet effort as scrub, circulator and recovery nurse in laparoscopic urological surgery. From the researchers clinical experience at operating room in Assiut urology and Nephrology hospital it has been observed that the nurses working in operating room lack knowledge regarding laparoscopy procedure preparations, so this study targeted those nurses in an attempt to raise their knowledge and skills toward laparoscopy procedure. The aim of this study was to assess the effect of nursing intervention protocol about laparoscopic urological surgery on nurse's performance and patient outcome through:

- Assessing nurse's knowledge and practice regarding laparoscopic surgery. 
- Developing and implementing nursing intervention protocol for patients undergoing laparoscopic surgery based on nurses needs assessment.

- Evaluate the effect of applying the designed nursing intervention protocol on nurse's performance and patient outcome.

\section{Research Hypotheses}

The following research hypotheses will be formulated:

1. The mean knowledge score of nurse's will be higher after the application of the nursing intervention protocol in laparoscopic surgery in urology.

2. The practice level of nurse's will be better after the application of the nursing intervention protocol in laparoscopic surgery in urology.

3. Outcomes of patient will be better the application of nursing intervention protocol in laparoscopic surgery in Uno fogy.

Operational definition about patients outcomes as reducing operation duration time and patient complications (shoulder pain, Burns from electric current were one of the major causes of complications during laparoscopic surgery and hyprcarbia)

\section{Subjects \& Methods \\ Research design}

Quasi experimental (pre-posttest) research design was used to conduct this study. Setting:

The study was carried out in the urological operating room at AssiutUrology and Nephrology Hospital from $20 / 5 / 2017$ to $20 / 5 / 2018$.

\section{Sample}

The sample consisted of two groups:

Group (1) All the available nurses (17) who scrub or circulate in the laparoscopic urological operating room.

Group (2) a purposeful sample of patients (60) who underwent laparoscopic surgery: 30 patients pre application of the nursing intervention protocol and 30 patients post application of the nursing intervention protocol. This sample was selected based on power analysis of $80 \%$ and the required sample $5 \%$ was determined using (Epi info software).

Tools of data collection:

Tool I: Pre/post nurse's knowledge assessment questionnaire sheet:

This tool was developed by the researcher after reviewing the literature and included two parts:

Part 1: demographic data of nurses (level of education, years of experience, training courses attendance).
Part2: Assessment of nurses 'level of knowledge regarding laparoscopic surgery as definition, indications, complications, nursing intervention, preoperative nursing care, intraoperative nursing care and post-operative nursing care. Scoring system: Each correct answer was given one score, the number of questions was 16. The total score of the questionnaires was less than $(60 \%)$ were considered unsatisfactory level. While those who obtained $(60 \%)$ or above were considered satisfactory according to the range of the total scores, it laid between (0-50 degrees).

Tool II: Pre/post nurse's practice observational checklist:

This tool was developed by the researcher after reviewing the literature and was used to assess nurses' practice during laparoscopic surgery including preparing, assembling, handling laparoscopic surgery instruments principles of sterilization and disinfection of laparoscopy set. Regarding observational checklist: Scores assigned to each item are between 0 and 1 points as follows; $0=$ not done, and $1=$ done correct. According to range of total scores are (76marks). Nurse was classified as inadequate practice if her total score was $<70$, and was classified as adequate practice if her total score was $>70 \%$.

Tool III: The designed nursing intervention protocol:

It was developed based on the analysis of pre assessment stage as the patient basic identified need .A booklet was developed, revised and modified based on the expertise comments; it was written in Arabic simple language with illustrations regarding every aspect concerned with the urological laparoscopy surgery.

Tool IV: Patient assessment sheet:

It was developed by the researcher based on the literature review. It consisted of:

Part 1: demographic and medical data of the patient as patient's code, age, gender, marital status, diagnosis and allergy to any medication to assess the patient's profile.

Part 2: Evaluation of laparoscopic surgery patient outcomes to evaluate the effect of applying the designed nursing protocol.

\section{Methods}

- Official permission to conduct the study was obtained from the hospital responsible authorities in the operating room in urology after explaining the aim and the nature of the study.

- Tools of data collection were developed after reviewing the relevant national and international literature. 
- The study tools was tested for content validity by five experts in medical and medical-surgical nursing staff at Assiut Urology and Nephrology Hospital and faculty of nursing who reviewed the tools for clarity, understanding, applicability and easiness for administrative, minor modifications where required.

\section{Ethical considerations}

- Research proposal approved from ethical committee in the faculty of nursing.

- Assure that there is no risk for study subjects during application of the research.

- The study will follow common ethical principles in clinical research.

- Written consent will be obtained from patient's or guidance that are willing to participate in the study after explaining the nature and purpose of the study

- Confidentiality and anonymity will be assured.

- Study subject have the right to refuse to participate and / or to withdraw from the study without any rational and at any time

- Study subjects privacy will be considered during data collection of data.

\section{A pilot Study}

It was conducted on $10 \%$ of the sample to examine feasibility and clarity of the study tools. Those patient were included in the main study .because modification were needed.

- Field of work: the researcher started the study by developing the protocol through extensive literature review that was based on the effect of the nursing interventions protocol and it's results on nurses performance and patients out come about the laparoscopic procedure.

- Preparation phase: a questionnaire on the extent of knowledge and practice of the seventeen nurses with laparoscopic procedure, preparation of machines, diathermy, suction, and laparoscopic tower, sterilization and preparation of the operating room by using tools I, II.

- Implementation phase: The researcher began distributing the booklet containing information about the ideal practice for nursing in urologic laparoscopic surgery, machines used and patient preparation which were explained in several sessions.

- The first session which ranged between 20- 40 minutes and its contents were; the meaning of the laparoscopy abdominal surgery, its advantages and disadvantages, complications and devices used in the operation.

- The second session took between 20-30 minutes and it included information about light source and camera, the difference between the cylinder of
$\mathrm{CO}_{2}$ and $\mathrm{O}_{2}$ and the amount of $\mathrm{CO}_{2}$ used for inflating the abdomen in adults and children.

- The third session ranged between 30-40 minutes consumed on the training of nurses on sterilization of the patient's skin and connection devices, machines and how to handle instruments to the surgeon.

- As for the fourth and last session, it ranged between 20-30 minutes where the nurses were trained by the researcher on instruments washing, disinfection and sterilization. Also they were trained on the cleaning of the operating room and preparing it to receive the next patient.

- Evaluation phase :The same questionnaire was distributed once again after applying the booklet to find out the extent to which the nurses were able to receive the training information and correspondence of laparoscopic surgical by using the first and second tools.

- A sample of 60 adult patients was enrolled in the study; 30 patients before the application of the protocol and 30 patients after the application of the protocol to examine the effect of this study on patients' outcomes.

- The means age of the studied patient sample was (22) years, the majority of them were working and all of them were male and single.

- Patients who were enrolled in this study were assessed by use of patient assessment sheet (tool IV).

- Both groups were semilar in there demographic characteristic .

Statistical analysis

The data collected was coded and analyses using the Statistical Package for Social Sciences (SPSS) version 23 for Windows. Descriptive statistics was used for the quantitative data in the all questionnaire and the demographic data. Descriptive statistics included: frequencies, and percentages. Use Pearson chi-square between nurses knowledge and sociodemographic data were done. The level of significance for this study was set at $(p \leq 0.05)$ to detect any indication of differences found in the data available. 


\section{Results}

Table (1): Percentage distribution of demographic data among the nurses.

\begin{tabular}{|c|c|c|c|}
\hline Variables & & Number(17) & $\%$ \\
\hline \multicolumn{4}{|c|}{ Level of education: } \\
\hline • & Secondary nursing school & 2 & 11.8 \\
\hline$\overline{\mathrm{N}}$ & Institute & 9 & 52.9 \\
\hline$\bullet$ & college & 6 & 35.3 \\
\hline \multicolumn{4}{|c|}{ Years of experience: } \\
\hline - & $<3$ years & 8 & 47.1 \\
\hline$\bullet$ & 3 years- 5 years & 3 & 17.6 \\
\hline$\cdot$ & $>5$ years & 6 & 35.3 \\
\hline \multicolumn{4}{|c|}{ Training attendance: } \\
\hline • & Yes & 11 & 64.7 \\
\hline$\bullet$ & No & 6 & 35.3 \\
\hline
\end{tabular}

Table (2): Comparison between total nurse's level of knowledge pre and post application of the nursing intervention protocol regarding laparoscopic surgery. $(n=17)$.

\begin{tabular}{|l|c|c|c|c|c|}
\hline \multirow{2}{*}{\multicolumn{1}{|c|}{ Variables }} & \multicolumn{2}{|c|}{ Pre test } & \multicolumn{2}{c|}{ Post-test } & \multirow{2}{*}{ P. value } \\
\cline { 2 - 5 } & N. & $\%$ & N. & $\%$ \\
\hline Total knowledge & & & & \multirow{2}{*}{$.001^{* * *}$} \\
\hline - Satisfactory. & 4 & 23.5 & 16 & 94.1 & \\
\hline - Unsatisfactory. & 13 & 76.5 & 1 & 5.9 & \\
\hline
\end{tabular}

Chi-Square test $* *=$ highly significance $* \mathrm{p} \leq 0.01$

Table (3): Nurses practice in Preoperative phase (in Receiving area) pre and post application of the nursing intervention protocol.

\begin{tabular}{|c|c|c|c|c|c|c|c|c|c|}
\hline \multirow{3}{*}{ Steps } & \multicolumn{4}{|c|}{ Pre-test } & \multicolumn{4}{|c|}{ Post-test } & \multirow{3}{*}{ p. value } \\
\hline & \multicolumn{2}{|c|}{ Done } & \multicolumn{2}{|c|}{ Not done } & \multicolumn{2}{|c|}{ Done } & \multicolumn{2}{|c|}{ Not done } & \\
\hline & $\mathbf{N}$ & $\%$ & $\mathbf{N}$ & $\%$ & $\mathbf{N}$ & $\%$ & $\mathbf{N}$ & $\%$ & \\
\hline 1.Introduces self to patient & 17 & 100 & 0 & $\overline{0}$ & 17 & 100 & 0 & 0 & $\begin{array}{c}----- \\
-1\end{array}$ \\
\hline $\begin{array}{l}\text { 2.Ask patient to undress and change into } \\
\text { operating room gown }\end{array}$ & 17 & 100 & 0 & 0 & 17 & 100 & 0 & 0 & ----- \\
\hline 3. Hand washing & 16 & 94.1 & 1 & 5.9 & 17 & 100 & 0 & 0 & 0.50 \\
\hline $\begin{array}{l}\text { 4.Place a bracelet on patient wrist with patient } \\
\text { name }\end{array}$ & 12 & 70.6 & 5 & 29.4 & 17 & 100 & 0 & 0 & $0.02 *$ \\
\hline $\begin{array}{l}\text { 5. An allergy bracelet will be placed on patient } \\
\text { wrist if applicable. }\end{array}$ & 12 & 70.6 & 5 & 29.4 & 17 & 100 & 0 & 0 & $0.02 *$ \\
\hline $\begin{array}{l}\text { 6. Talk through the events of the day, allow } \\
\text { patient to ask any questions and check for } \\
\text { informed consent }\end{array}$ & 16 & 94.1 & 1 & 5.9 & 17 & 100 & 0 & 0 & 0.50 \\
\hline 7. Check that all test results are available. & 14 & 82.4 & 3 & 17.6 & 17 & 100 & 0 & 0 & 0.11 \\
\hline 8. Ask about last time patient ate and drank & 15 & 88.2 & 2 & 11.8 & 17 & 100 & 0 & 0 & 0.24 \\
\hline $\begin{array}{l}\text { 9. If patient wear dentures, wig, spectacles or } \\
\text { hearing aid these can be left in place }\end{array}$ & 16 & 94.1 & 1 & 5.9 & 17 & 100 & 0 & 0 & 0.50 \\
\hline $\begin{array}{l}\text { 10. Contact lenses, jewelers, make up and nail } \\
\text { polish will be removed }\end{array}$ & 16 & 94.1 & 1 & 5.9 & 17 & 100 & 0 & 0 & 0.50 \\
\hline 11.Measure patient vital signs & 16 & 94.1 & 1 & 5.9 & 17 & 100 & 0 & 0 & 0.50 \\
\hline Total & 15 & 88.2 & 2 & $\mathbf{1 1 . 8}$ & 17 & $\overline{100}$ & & & $0.0^{*}$ \\
\hline
\end{tabular}

Chi-Square Tests ${ }^{*}=$ Significant difference, ${ }^{*} p \leq 0.0$

$N s=$ Non significant difference $\quad p<0.05$ 
Table (4): Nurses practice in intra operative phase pre and post application of the nursing intervention protocol.

\begin{tabular}{|c|c|c|c|c|c|c|c|c|c|}
\hline \multirow{3}{*}{ Circulating nurse's role } & \multicolumn{4}{|c|}{ Pre-test } & \multicolumn{4}{|c|}{ Post-test } & \multirow{3}{*}{$\begin{array}{c}\text { P. } \\
\text { Value }\end{array}$} \\
\hline & \multicolumn{2}{|c|}{ Done } & \multicolumn{2}{|c|}{ Not done } & \multicolumn{2}{|c|}{ Done } & \multicolumn{2}{|c|}{ Not done } & \\
\hline & $\mathbf{N}$ & $\%$ & $\mathbf{N}$ & $\%$ & $\mathbf{N}$ & $\%$ & $\mathbf{N}$ & $\%$ & \\
\hline $\begin{array}{l}\text { 1. Ensures Sterile environment and all } \\
\text { supplies needed are available. }\end{array}$ & 16 & 94.1 & 1 & 5.9 & 17 & 100 & 0 & 0 & 0.50 \\
\hline $\begin{array}{l}\text { 2. Monitor and document the supplies } \\
\text { taken during the operation. }\end{array}$ & 16 & 94.1 & 1 & 5.9 & 17 & 100 & 0 & 0 & 0.50 \\
\hline $\begin{array}{l}\text { 3. Function to promote the sterility of } \\
\text { the operating room. }\end{array}$ & 16 & 94.1 & 1 & 5.9 & 17 & 100 & 0 & 0 & 0.50 \\
\hline $\begin{array}{l}\text { 4. Informing operating staff of anything } \\
\text { causes contamination. }\end{array}$ & 15 & 88.2 & 2 & 11.8 & 17 & 100 & 0 & 0 & 0.24 \\
\hline $\begin{array}{l}\text { 5. Responsible for opening autoclaved } \\
\text { packages. }\end{array}$ & 17 & 100 & 0 & 0 & 17 & 100 & 0 & 0 & -- \\
\hline $\begin{array}{l}\text { 6. Make sure the } \mathrm{CO}_{2} \text { cylinder full not } \\
\text { empty or not broken. }\end{array}$ & 12 & 70.5 & 5 & 29,4 & 17 & 100 & 0 & 0 & $0.02 *$ \\
\hline $\begin{array}{l}\text { 7. Receive the patient form recovery } \\
\text { room and his file. }\end{array}$ & 13 & 76.5 & 4 & 23.5 & 17 & 100 & 0 & 0 & $0.05 *$ \\
\hline $\begin{array}{l}\text { 8. Ensure that test results and x-rays are } \\
\text { available in patient file. }\end{array}$ & 15 & 88.2 & 2 & 11.8 & 17 & 100 & 0 & 0 & 0.24 \\
\hline $\begin{array}{l}\text { 9. Check for patient consent form for } \\
\text { the operation. }\end{array}$ & 13 & 76.5 & 4 & 23.5 & 17 & 100 & 0 & 0 & $0.05 *$ \\
\hline $\begin{array}{l}\text { 10. Making sure that all operating } \\
\text { instrument is counted. }\end{array}$ & 13 & 76.5 & 4 & 23.5 & 17 & 100 & 0 & 0 & $0.05 *$ \\
\hline 11. Help in positioning the patient. & 13 & 76.5 & 4 & 23.5 & 17 & 100 & 0 & 0 & $0.05^{*}$ \\
\hline Total & 15 & 88.2 & 2 & 11.8 & 17 & 100 & $\mathbf{0}$ & $\mathbf{0}$ & $0.0 *$ \\
\hline
\end{tabular}

Chi-Square Tests *=Significant difference,

$*_{p} \leq 0.05, * *=$ highly significance, $*_{p} \leq 0.01 \quad N_{s}=$ Non significant difference $\quad \mathrm{p}<0.05$.

Table (5): Nurses practice in post-operative care phase pre and post application of the nursing intervention protocol.

\begin{tabular}{|c|c|c|c|c|c|c|c|c|c|}
\hline \multirow{3}{*}{ post-operative nursing care phase } & \multicolumn{4}{|c|}{ Pre-test } & \multicolumn{4}{|c|}{ Post-test } & \multirow{3}{*}{$\begin{array}{c}\mathrm{p} . \\
\text { value }\end{array}$} \\
\hline & \multicolumn{2}{|c|}{ Done } & \multicolumn{2}{|c|}{$\begin{array}{c}\text { Not } \\
\text { done }\end{array}$} & \multicolumn{2}{|c|}{ Done } & \multicolumn{2}{|c|}{$\begin{array}{c}\text { Not } \\
\text { done }\end{array}$} & \\
\hline & $\mathbf{N}$ & $\%$ & $\mathbf{N}$ & $\%$ & $\mathbf{N}$ & $\%$ & $\mathbf{N}$ & $\%$ & \\
\hline $\begin{array}{l}\text { 1. Checking the patient's airway and } \\
\text { breathing. }\end{array}$ & 14 & 82.4 & 3 & 17.6 & 17 & 100 & 0 & 0 & 0.11 \\
\hline 2. Pain relief managements. & 16 & 94.1 & 1 & 5.9 & 17 & 100 & 0 & 0 & 0.50 \\
\hline $\begin{array}{l}\text { 3. Shift patient from the recovery room to } \\
\text { the inpatient department. }\end{array}$ & 16 & 94.1 & 1 & 5.9 & 17 & 100 & 0 & 0 & 0.50 \\
\hline $\begin{array}{l}\text { 4. Provide patient support, instructions } \\
\text { needed for home care. }\end{array}$ & 15 & 88.2 & 2 & 11.8 & 17 & 100 & 0 & 0 & 0.24 \\
\hline 5.Monitor the patient condition & 15 & 88.2 & 2 & 11.8 & 17 & 100 & 0 & 0 & 0.24 \\
\hline $\begin{array}{l}\text { 6. Vital signs and saturation taken and } \\
\text { recorded every } 15 \mathrm{~min} \text {. }\end{array}$ & 16 & 94.1 & 1 & 5.9 & 17 & 100 & 0 & 0 & 0.50 \\
\hline 7. Monitor oxygen saturation. & 17 & 100 & 0 & 0 & 17 & 100 & 0 & 0 & -- \\
\hline 8. Record Urine output. & 17 & 100 & 0 & 0 & 17 & 100 & 0 & 0 & -- \\
\hline 9. Assessment of wound sites. & 14 & 82.4 & 3 & 17.6 & 17 & 100 & 0 & 0 & 0.11 \\
\hline $\begin{array}{l}\text { 10. Replacing intravenous fluids and } \\
\text { reporting any abnormalities. }\end{array}$ & 17 & 100 & 0 & 0 & 17 & 100 & 0 & 0 & -- \\
\hline Total & 16 & 94.1 & 1 & 5.9 & 17 & 100 & 0 & 0 & $0.0^{*}$ \\
\hline
\end{tabular}

Chi-Square Tests $\quad N s=$ Non significant difference $p<0.05$ 
Table (6): Percentage distribution of laparoscopic intervention needed for the studied patients.

\begin{tabular}{|l|c|c|}
\hline \multicolumn{1}{|c|}{ Variables } & Number & $\%$ \\
\hline Diagnostic laparoscopy. & 22 & 36.7 \\
\hline Nephrectomy by laparoscopy. & 10 & 16.7 \\
\hline Rt.deroofing of simple renal cyst. & 10 & 16.7 \\
\hline Pyeloplasty. & 6 & 9.9 \\
\hline Rt. abdominal test is with1st and 2nd stage previously. & 10 & 16.7 \\
\hline Left impalpable test with distal penil hypospadias. & 2 & 3.3 \\
\hline Total & 60 & 100.0 \\
\hline
\end{tabular}

Table (7) : Comparison between pre and post-test groups regarding patient complications.

\begin{tabular}{|c|c|c|c|c|c|c|c|c|c|}
\hline \multirow{3}{*}{ Variables } & \multicolumn{4}{|c|}{ Pretest ( group $n=30)$} & \multicolumn{4}{|c|}{ Posttest ( group $n=30)$} & \multirow{3}{*}{ P.value } \\
\hline & \multicolumn{2}{|c|}{ Present } & \multicolumn{2}{|c|}{ No present } & \multicolumn{2}{|c|}{ Present } & \multicolumn{2}{|c|}{ No present } & \\
\hline & $\mathbf{N}$ & $\%$ & $\mathbf{N}$ & $\%$ & $\mathbf{N}$ & $\%$ & $\mathbf{N}$ & $\%$ & \\
\hline Signs of infection. & 8 & 26.6 & 22 & 73.3 & 2 & 6.7 & 28 & 93.3 & $0.040 *$ \\
\hline Stomach pain. & 6 & 20.0 & 24 & 80.0 & 0 & 0 & 30 & 100 & $0.01 *$ \\
\hline Chills. & 9 & 30.0 & 21 & 70.0 & 3 & 10.0 & 27 & 90.0 & $0.05^{*}$ \\
\hline Nausea or vomiting. & 4 & 13.3 & 26 & 86.7 & 0 & 0 & 30 & 100 & $0.05^{*}$ \\
\hline Persistent cough. & 0 & 0 & 30 & 100 & 0 & 0 & 30 & 100 & --- \\
\hline Shortness of breath. & 0 & 0 & 30 & 100 & 0 & 0 & 30 & 100 & --- \\
\hline Inability to urinate. & 12 & 40.0 & 18 & 60.0 & 4 & 13.3 & 26 & 86.7 & $0.02 *$ \\
\hline Light headedness. & 3 & 10.0 & 27 & 90.0 & 0 & 0 & 30 & 100 & 0.119 \\
\hline Shoulder pain. & 14 & 46.7 & 16 & 53.3 & 3 & 10 & 27 & 90.0 & $0.002 * *$ \\
\hline
\end{tabular}

Table (8): Relation between total nursing practices mean score pre and post application of the nursing intervention protocol and correlation between total knowledge and total practice scores of the nurses.

\begin{tabular}{|l|c|c|}
\hline \multirow{2}{*}{\multicolumn{1}{c|}{ Variables }} & Total practice score & \multirow{2}{*}{ P. value } \\
\cline { 2 - 3 } & Mean \pm SD & \multirow{2}{*}{$0.001^{* *}$} \\
\hline Pretest & $13.25 \pm 140.41$ & \\
\hline Posttest & $127.00 \pm .00$ & \\
\hline & Pearson Correlation & $.027^{*}$ \\
\hline
\end{tabular}

Independent $t$-test and Pearson correlation positive $p \leq 0.05$ used for this table.

Table (1): Showed that regarding education the highest percent of the nurses $(52.9 \%)$ were having a nursing institute education, regarding years of experience $(47.1 \%)$ of the nurses were less than 3years and $(64 \%)$ of them attended training courses Table (2): Showed that there was a highly statistically significant difference between nurses knowledge about laparoscopy pre and post application of the nursing intervention protocol.

Table (3): Showed that in the pre-laparoscopic surgery nursing care of the patient. we can see that there was a statistically significant difference pre and post application of the nursing intervention protocol regarding placing bracelet or An allergy bracelet were placed on patient wrist for the patient with his /her name and hospital number ( $\mathrm{p}$-value $=0.02$ ),

Table (4): Showed that regarding the circulating nurse's role pre \& post application of the nursing intervention protocol; a statistically significant difference was found between the following items; receiving the patient form recovery nursing and patient's file, checking for patient consent form for the operation, making sure that all operating equipment and supplies is counted for before and after the operation together with the scrub nurse and making sure the $\mathrm{CO}_{2}$ cylinder is full and not empty .

Table (5): Showed that there was no statistically significant difference regarding post-operative nursing care pre and post application of the nursing intervention protocol

Table (6): Showed that regarding diagnosis of the patients, the highest percentage underwent Laparoscopic surgery were $(36.7 \%)$ for diagnostic purposes. of infection control.

Table (7): Showed that there was a statistically significant difference between both times of follow- 
up regarding signs of infection, stomach pain, chills, nausea and vomiting, patient inability to urinate and shoulder pain after the procedure.

Table (8): Showed that there was a highly statistically significant difference between pre and post application of the nursing intervention protocol. Regarding total nursing practice mean score reveals a positive correlation between nurses' knowledge and practice regarding urological abdominal laparoscopic surgery

\section{Discussion}

Laparoscopic surgery caused a major turning point in surgery during recent years used by surgeons. Laparoscopic surgery has become a popular approach for a number of procedures previously performed openly Nomi, et al., (2015).

The aims of this study were to assess nurse's knowledge and practice regarding laparoscopic surgery, Design a nursing intervention protocol for patients undergoing laparoscopic surgery, and evaluate the effect of applying the designed nursing intervention protocol on nurse's performance and patient outcome.

Findings of this study regarding nurse's knowledge pre and post application of the nursing intervention protocol about laparoscopic procedure there was a highly statistically significant difference between nurses knowledge about laparoscopy pre and post application of the nursing intervention protocol. Nearly all nurses were not having the correct answer for questions regarding patient's age for laparoscopic surgery, the suitable abdominal pressure for insufflation during laparoscopic surgery and the level of knowledge among the majority of nurses about the laparoscopic surgery .

Bhagirathee, (2013) study told that Knowledge about laparoscopic surgery varied amongst the theatre and the ward nurses to be knowledgeable about laparoscopic surgery. However, specific limitations in the nurses' knowledge were identified. Also stated Although most of the theatre nurses were mature in age and have been in nursing for a considerable number of years, it is a concern to note that only about half of them were knowledgeable about laparoscopic surgery and this agree with the present study.

According to (Pera \& Van Tonder, 2011) the reasons for a nurse to act as a patient's advocate is for the quality of care that the patient receives, the patient's access to care, the patient's awareness of the care, its effects and side effects and the patient's understanding of the alternatives to the proposed treatment. Witnessing a patient giving consent to an operation by a nurse implies accountability on the part of the nurse, hence the importance of being knowledgeable about the laparoscopic procedure surgery.

This study stated that the efficient nursing care is important during recovery period. Nurses must be prepared to prevent postoperative complications, rather than waiting to treat them. Nurses can provide excellent care if they are able to anticipate a patient's needs provide early intervention when symptoms first appear early provide reassurance to alleviate patients' uncomfortable feeling during the recovery process, and educate patients to alleviate unnecessary anxiety related to discharge expectations. And this lined with the (International Journal of Medical \& Health Research May 2016).

In the present study, it was clear that the training program had positive effects in improving the nurses' performance as regard nursing care of patients, definition, types, contraindications, preparation the site of surgery general instructions, exercise, and wound care. This was proved by the significant difference between results of the pre-test and post-test program implementation. This indicates that skills are easier to improve, especially if linked with their relevant scientific basic information and needs. The present study result reveled that there are statistically significant differences was found between nurses knowledge about laparoscopic surgery pre \& post application of the nursing intervention protocol.

Phillips, (2007) confirms that due to the intricacies of the instrumentation and the limits of the visual field strict adherence to details and astute anticipation during the surgery is required hence the need for adequate knowledge for the nurses and these agree with the present study.

Regarding the total nursing practice there was a highly statistically significant difference between pre and post application of the nursing intervention protocol practice.

The present study reveals a positive correlation between nurses' knowledge and practice regarding urologic abdominal laparoscopic surgery.

The present study results agree with Carol, (2009) who stated that training increases the perioperative nurse's knowledge about the procedures and provides direct experience and appreciation of the activities at the sterile field. This gives the nurse greater competence at delegating their role, as well as to lean skill that requires experience as well as effective communication.

The results of the present study found that the nurses does not play her role regarding checking if the consent form present or not. Nurses are not advocates for patients meaning that they need to ensure that they are aware of his or her rights and in the case of the consent taking. This comes in line with Bhagirathee, (2013) as in study who stated it 
appears that the health professional team including the doctors and nurses are at times negligent about 120 informed consent signing in the case of laparoscopic surgery, as the omission of conversion to open surgery is a serious medico-legal issue.

The researcher stressed in this study on the intra operative phase and the essential role of the nurses during laparoscopic surgery and its reflection on patient's outcomes. This is in line with Jenkins, (2013) when they stated that experienced scrub nurses can markedly decrease surgery time due to their knowledge and understanding, resulting in a swifter turnover between procedures and a high level of patient safety.

The results from this study revealed that the professional nurses who participated in this study were not sufficiently knowledgeable about intra operative phase laparoscopic surgery. Bonjer, (2015) also agreed with the present study by stating that nurses who provide postoperative care must have knowledge of the implications of the procedure, clinical manifestations of complications, and risk factors. Identifying patients at high risk for adverse outcomes allows the nurse to anticipate the needs of the patient and provide a less stressful postoperative experience.

The Bhagirathee, (2013) study found that the theatre nurses were better informed about the intra-operative complications of laparoscopic surgery but the theatre did not perceive it as safe. Although they were aware of the advantages of laparoscopic surgery, they still preferred open surgery themselves. This could possibly be attributed to the fact that they were afraid of the many possible intra-operative complications due to the instrumentation and also of advanced technology expectations. The present study lined with Bhagirathee, (2013) study regarding that the nurse's preferences to scrub in open surgery rather than the laparoscopic surgery.

The result of the present study revealed that there is a positive correlation between nurse's knowledge and practice regarding urologic abdominal laparoscopic surgery. This agreed with Liddle, (2013) as he is in line with the finding that the nurses who provide postoperative care must have satisfactory level of knowledge about the implications of the procedure.

There was a statistically significant difference pre and post application of the nursing intervention protocol regarding nursing care provided to patients. These results are in line with the Palmer et al., (2013) study those who found that the layout of the OR the circulating nurses is the most ambulatory surgical team member and potentially impacted by and involved in a range of flow disruptions. However, no studies have explored how flow disruptions may impact the work of the circulating nurses. An operating room layout can influence work patterns of circulating nurses.

Bhagirathee, (2013) reported that nurses improved outcomes reflects on patients in that there were less blood loss, and lower complications rates. The standards of nursing care for patients recovering from laparoscopic urology are comprehensive and including monitoring, evaluating, and followed by treating of the postoperative care of patients with such surgery .

When we look at the demographic data of patients we can see that the mean age \pm standard deviation of the studied sample was $(21.79 \pm 2.19)$ years, the majority of them were working and all of them were male and single.

Regarding the comparison between the studied patients' pre and post applications of the nursing intervention protocol; there was a statistically significant difference between both groups at the times of follow-up regarding signs and symptoms of infection, stomach pain, chills, nausea and vomiting, patient inability to urinate and shoulder pain after the procedure. This study comes with the study of

Koutoukidis et al., (2017) they stated there are many factors which can contribute to decreased urinary function following surgery, resulting in urinary retention. These include pain, anxiety and a depressed micturition reflex with certain anaesthetic agents and by nursing Management of urinary retention includes encouraging ambulation, assuming a normal voiding position, ensuring adequate fluid intake, double voiding and providing sensory stimulation such as running water when trying to void, and as a last resort, catheterization

The present study clarifies that most of the nurse oriented about how to make Assessment of wound sites. The present study show that the most of the nurses. Checking the patient's airway and breathing pattern and Pain relief managements.

Hussein, (2018) study agrees with the present study about hypothesis that patient who practice-breathing exercises had a better quality of recovery than those who do not. Gradually increased activities are necessary to prevent postoperative complications. Analgesics should be given as prescribed and the patient assisted to turn, cough, and breathe deeply.

Present study comes with Bhagirathee, (2013) finding regarding Nurses needed to monitor postoperative nausea and vomiting, wound sites and drains.

Also Graham, (2008) study he states that postoperative nausea and vomiting is common because of peritoneal insufflation and the manipulation of the bowel. The use of opioids which 
is a common cause of post-operative nausea and vomiting should be kept to

LingboPeng, (2015) stated that although perioperative nursing of urology is complicated, as long as the nursing staff develops the habit of carefulness and rigorousness, have the attitude of evidence- based medicine, continuously strengthen business study and summarize the work experience, they can timely discover and deal with the problems. These reduce the occurrence and development of the complications, improve the success rate of the urological laparoscopic procedure, reduce the pain of patients and help patients recover as early as possible. Meticulous, careful and comprehensive perioperative nursing plays an important positive role in the early recovery of the patients

Finally this study found that the nurses have no idea regarding assessing of the $\mathrm{CO} 2$ cylinder if it is empty or full or how to differentiate between the $\mathrm{O} 2$ cylinder and $\mathrm{CO} 2$ cylinder and how to operate the insufflators there are no studies talks about this issue

\section{Conclusion}

Based on the present study, it can be concluded that

- There was a statistically significant difference between nurses' knowledge and practice about laparoscopy pre and post application of the nursing intervention Protocol because the nurses' knowledge and understanding about laparoscopic surgery was limited.

- Patients included in the study post application of the nursing intervention protocol had lower incidence of complications than those patients who were included pre application of the nursing intervention protocol.

\section{Recommendations}

Based on the results of the present study we recommend that

- Encourage nurses to attend scientific meetings, conferences and workshops to improve their knowledge and level of professional performance.

- A well-structured training programs should be offered to the nurses to be well prepared, feel confident, and maintain high-quality of care and to keep them pace with the rapidly growing wealth of knowledge and practice.

- The nursing intervention protocol is to be applied in different health care urology settings for generalization of the data.

\section{Reference}

1. Bonjer H., Deijen C., Abis G., Cuesta M., Martjin H., (2015): "A randomized trial of laparoscopic versus open surgery for rectal cancer." The New England Journal of Medicine.;
372:1324-1332.

http://www.nejm.org/doi/full/10.1056/NEJMoa14 14882\#t=article International Journal of Medical and Health Research May 2016.

2. Carol. R., (2009): Ritchie MSN, RN, CNORcritchie@shc.org Perioperative Nursing Clinics Volume 4, Issue 2, June 2009, Pages 167180 Scottsdale Healthcare, 7400 East Osborn Road, Scottsdale, AZ 85251, USA

3. Palmer, J., Abernathy, G., Swinton, D., Allison, J., Greenstein, S., Shappell, K., Juang, S., (2013): Reeves Realizing improved patient care through human-centered operating room design: a human factors methodology for observing flow disruptions in the cardiothoracic operating room Anesthesiology, 119 (5) (2013), pp. 1066-1077

4. Hussein, E., \& Taha, N., (2018): Medical Surgical Nursing, Faculty of Nursing, Zagazig University,

5. Jenkins, H., (2013): RVN, FdSc, uses the case study of a German shepherd dog undergoing total hip replacement to look at the skills needed by a nurse assisting in theatre.

6. Kang, M, Gagne C., De, H., Kang (2016): Perioperative nurses' work experience with robotic surgery: a focus group study Comput Inf Nurs, 34, pp. 152-158.

7. Koutoukidis, G., Stainton, K., \& Hughson, J., (eds) (2017): Tabbner's Nursing Care: Theory and Practice, 7 th edn, Elsevier, Chatswood

8. Liddle C., (2013): postoperative care 1:principles of monitoring postoperative patient .Nursing Times :109:22,24-26 Birmingham City University Abstract.

9. LingboPeng (2015): Journal of Nursing (Volume 4Issue :December 2015).indd

10. Nomi, T., Fuks, D., Agrawal, A., Kawaguchi, Y., Ogiso, S., Gayet, B., (2015): Totally laparoscopic right hepatectomy combined with resection of the inferior vena cava by anterior approach. Ann. Surg Oncol. 22 (3), 851.

11. Pera, S., \& Van Tonder, S., (2011): Ethics in health care. 3rd edition. Cape Town: Juta

12. Phillips, N., (2007): Berry and Kohn's operating room technique. 11th edition. St Louis: Mosby.

13. Pravina Devi Bhagirathee (2013): university of South Africa supervisor: professor e potgieter june 2013

14. Thomas, C., Francis. P., (2011): win field HN.Urol.Nurs. -Role of the perioperative nurse in robotic surgery Perioper Nurs Clin, 6, pp. 227232006 Apr; $\quad 26$

(2):110-5. Review.PMID:16703918 